\title{
AVALIAÇÃO DA SAÚDE BUCAL DE IDOSOS POR ENFERMEIROS: VALIDADE E CONFIABILIDADE DO INSTRUMENTO ASBTO
}

\author{
Ana Lúcia Schaefer Ferreira de MELLO ${ }^{a}$, Karoline ZIMERMANN \\ Lucia Hisako Takase GONÇALVES ${ }^{c}$
}

\section{RESUMO}

O objetivo do estudo foi aplicar o Instrumento "Avaliação da Saúde Bucal para Triagem Odontológica” (ASBTO) em idosos institucionalizados para determinação dos índices de validade e confiabilidade, quando da aplicação por Enfermeiros. Os exames foram executados por um Enfermeiro e por um Cirurgião-Dentista (CD) em 50 idosos de uma instituição de longa permanência. A determinação da consistência interna foi verificada por meio do Coeficiente Alfa de Cronbach e ANOVA. Para determinação da estabilidade e confiabilidade foram considerados os percentuais de concordância e teste Kappa. Não houve diferença estatística significante entre as médias finais obtidas pelo CD e pelo Enfermeiro $(p=0,41)$. Houve maior consistência interna nos exames procedidos pelo CD. Os percentuais de concordância variaram de 60\%, na categoria Lábio, até 90\%, na categoria "Dentes naturais". O Kappa total do instrumento alcançou 0,46. A utilização do ASBTO é passível por Enfermeiros. Faz-se necessária adequada capacitação e treinamento prático para padronização de critérios.

Descritores: Idoso. Saúde Bucal. Instituição de Longa Permanência para Idosos.

\section{RESUMEN}

Se objetivó aplicar el Instrumento Evaluación de la Salud Bucal (IESB) para la determinación de la fiabilidad y validez, cuando de la aplicación por enfermeros. Los exámenes fueron ejecutados por un Enfermero y por un Dentista en 50 ancianos institucionalizados. La determinación de la consistencia interna se verificó a través del Coeficiente Alfa de Cronbach y test de ANOVA. Para la determinación de la estabilidad y fiabilidad fue considerado el percentil de acuerdo y prueba Kappa. No hubo diferencia estadística significante entre los promedios finales del Dentista y Enfermero $(p=0,41)$. Hubo más grande consistencia interna en los exámenes procedidos por el Dentista. Los porcentuales de concordancia entre Enfermero y Dentista variaron desde un 60\% - Labio, hasta un 90\% - Dientes. El valor Kappa total del instrumento alcanzó 0,46. El instrumento IESB puede ser utilizado por enfermeros para rastreo, sin embargo demanda entrenamiento práctico para ajuste de los criterios de examen.

Descriptores: Ancianos, Salud bucal. Hogares para ancianos.

Título: Evaluación de la salud bucal de ancianos por enfermeros: fiabilidad y validez del instrumento IESB.

\section{ABSTRACT}

The aim of the study was to apply the Oral Health Assessment Tool (OHAT) for determination of the reliability and validity indexes, when utilized by nursing staff. The OHAT was administered to 50 elderly individuals. The exams were performed in different periods of times by a Nurse and a Dental Surgeon $(D S)$. The determination of internal consistency was verified through Cronbach's Alpha Coeficient and ANOVA. For the determination of stability and reliability, the percentage agreement and Kappa test were considered. There was no statistical difference among the final averages obtained by the $D S$ and the nurse $(p=0.41)$. There was a higher internal consistency in the exams performed by the DS. The Kappa value of the instrument reached 0.46, being considered moderate. The OHAT instrument can be used by nurses as a screening tool; however, previous training is needed for criteria standardization.

Descriptors: Aged. Oral health. Homes for the aged.

Title: Nurse's assessment of oral health of elderly people: OHAT validity and reliability.

\footnotetext{
a Doutora em Enfermagem. Doutora em Odontologia em Saúde Coletiva. Professora do Departamento de Odontologia, Universidade Federal de Santa Catarina (UFSC), Florianópolis, Santa Catarina, Brasil.

b Bolsista Iniciação Científica, Conselho Nacional de Desencolvimento Científico e Tecnológico (CNPq/UFSC), Florianópolis, Santa Catarina, Brasil.

c Doutora em Enfermagem. Professora do Departamento de Enfermagem (UFSC), Florianópolis, Santa Catarina, Brasil.
} 


\section{INTRODUÇÃO}

Estudos realizados em instituições de longa permanência (ILPI) revelam que, neste contexto, o adequado cuidado com a saúde bucal não tem sido contemplado e costumeiramente observam-se flagrantes omissões ${ }^{(1-4)}$. No Brasil, não há normas específicas referentes ao cuidado à saúde bucal em instituições. Têm-se observado ausência de protocolos, falta de programação de atenção especializada, e ausência de programas de orientação e capacitação dos cuidadores profissionais ou leigos ${ }^{(5)}$.

À medida que as pessoas atingem as últimas décadas de vida, as necessidades de cuidados permanentes aumentam, inclusive em relação aos cuidados à saúde bucal. Dados apontam que a população idosa tende a apresentar uma precária condição de saúde bucal, em grande parte por reflexo de deficiências no cuidado à saúde bucal ao longo da vida. Os idosos que apresentam algum grau de dependência ou têm sua autonomia comprometida apresentam piores condições de higiene bucal ${ }^{(6-8)}$.

É reconhecida a necessidade do desenvolvimento e validação de instrumentos como ferramentas de screening a serem utilizadas por cuidadores em idosos que possuíssem algum grau de comprometimento cognitivo em ILPI, bem como de programas educativos-preventivos, estratégias comportamentais e de comunicação específicas para essa população ${ }^{(8)}$.

A saúde bucal de idosos residentes em instituições é bastante precária devido, principalmente, ao acesso limitado aos serviços odontológicos e à inexistência de uma rotina diária de higiene bucal. Porém, somente o acesso ao Cirurgião-dentista não é suficiente para solucionar os problemas de saúde bucal em ILPI ${ }^{(1)}$. A assistência odontológica para idosos institucionalizados apresenta-se como um enorme desafio: existência de pessoal treinado, disponibilidade de equipamentos, recursos para assistência e as condições de saúde geral e bucal dos idosos.

A prática de exames bucais em idosos residentes em ILPI deveria ser corrente, realizada pelos profissionais de enfermagem e cuidadores, no intuito de monitorar as condições de saúde bucal, avaliar a higiene bucal e, em casos de necessidade recorrerem a atendimento especializado. Para tanto, afirma-se a necessidade de formulação e validação de instrumentos elaborados para avaliar as condições de saúde bucal de idosos, a serem utilizados por profissionais de enfermagem e cuidadores em geral que sejam confiáveis, de fácil emprego nas rotinas diárias e que dispense recurso humano especializado.

A prática de validação de instrumentos para uso na área de Geriatria e Gerontologia tem se tornado freqüente no Brasil, tanto para a comunidade científica quanto para profissionais atuantes nos serviços de saúde para idosos ${ }^{(9-14)}$. $\mathrm{Na}$ área da saúde bucal do idoso, a literatura internacional também reporta validação de ferramentas ${ }^{(15,16)}$.

Este estudo pretendeu dar suporte a um estudo multicêntrico intitulado Instituições de Longa Permanência para Idosos - ILPIs no Brasil: tipologia e proposta de modelo básico de assistência multidimensional. Em se tratando de uma proposta de modelo multidimensional, incorporou-se o cuidado à saúde bucal, entendido como uma vertente do cuidado integral que os idosos recebem nestas instituições. Desse modo, a padronização e a validação de instrumentos a serem incluídos na implementação da avaliação diagnóstica multidimensional seguem a mesma linha de outros instrumentos que serão utilizados na avaliação das condições de vida e saúde dos idosos residentes como a Caderneta do Idoso do Ministério de Saúde, o índice de avaliação da capacidade funcional física da American Alliance for Health, Physical Education, Recreation and Dance (AAHPERD), as atividades físicas da vida diária pelo Índice de Katz, entre outros.

O objetivo deste estudo foi aplicar o Instrumento de Avaliação da Saúde Bucal para Triagem Odontológica em um grupo de idosos institucionalizados com vistas à determinação dos índices de confiabilidade e validade, quando da aplicação por profissionais de Enfermagem.

\section{MÉTODO}

\section{Tipo de Estudo}

Trata-se de um estudo transversal, de abordagem quantitativa, com vistas à validação de instrumento.

\section{O instrumento}

O instrumento Oral Health Assessment Tool $(O H A T)^{(16)}$ tem como objetivo fornecer aos cuidadores em ILPI um instrumento simples, com oito categorias de análise para acessar a saúde bucal dos residentes, inclusive aqueles com comprometimen- 
to cognitivo. Foi testado em 21 ILPI australianas, sendo reaplicado aos três e seis meses para testar a confiabilidade intra e inter cuidadores. $\mathrm{O}$ instrumento foi validado, apresentando reprodutibilidade e confiabilidade. Considerando a confiabilidade inter examinador, atingiu uma porcentagem desde 72,6\% para categoria Higiene bucal, até 92,6\% para Dor dental. O coeficiente kappa foi considerado moderado $(0,48$ a 0,60) para categorias Lábios, Língua, Gengiva, Saliva, Higiene bucal e para outras categorias foi de 0,61 a 0,80, classificadas como concordância substancial ${ }^{(15)}$.

A tradução e validação semântica do instrumento Oral Health Assessment Tool para o idioma português foi objeto de e publicação anterior. Ao final deste processo, passou-se a denominar Instrumento de Avaliação da Saúde Bucal para Triagem Odontológica (ASBTO) $)^{(17)}$.

Além da identificação do idoso, o instrumento possui 8 categorias independentes, que constituem os itens de análise (Lábios, Língua, Gengivas e Tecidos Moles, Saliva, Presença de Dentes Naturais, Presença de Dentaduras, Higiene Bucal e Dor).

Para cada item o cuidador deve atribuir um escore de 0 a $2(0=$ saudável, $1=$ presença de alterações ou 2 = não saudável), em função das condições bucais apresentadas pelo idoso. Ao final, somam-se as pontuações das 8 categorias obtendo-se a pontuação individual final. O escore final é a soma dos escores que pode então variar de 0 (muito saudável) a 16 (muito doente). Quanto maior o escore, pior a condição bucal do idoso. Uma vez que os pontos cumulativos são importantes para a avaliação da saúde bucal, a pontuação de cada item precisa ser considerada individualmente. Caso o idoso receba, em quaisquer dos itens, escore 1 ou 2 , é recomendado o encaminhamento do idoso para consulta odontológica.

\section{Coleta dos dados}

A aplicação do Instrumento ASBTO foi realizada em idosos residentes em uma ILPI, compondo uma amostra intencional. Como critérios de inclusão definiram-se: capacidade de compreensão dos objetivos do estudo e condição de saúde favorável para receber os procedimentos do exame clínico. Foram excluídos aqueles idosos que, por motivo de doença ou idade avançada, se apresentavam altamente fragilizados - condição que impedia o exame clínico. Participaram 50 idosos, com idade entre 60 a 98 anos.
Os idosos foram examinados individualmente em cadeiras, nos leitos ou na enfermaria da instituição. Utilizou-se espátula de madeira e luz natural. O Instrumento foi aplicado por um profissional graduado em Enfermagem e um em Odontologia, em seqüência aleatória e em horários ou datas distintas (nesse caso em menos de 48 horas de intervalo). Os dados foram anotados em formulários independentes. O Cirurgião-dentista não teve acesso aos dados coletados pelo Enfermeiro e vice-versa. Os idosos possuíam conhecimento sobre a profissão de cada examinador. Ambos profissionais receberam orientações idênticas e foram treinados para a aplicação do Instrumento.

\section{Confiabilidade Interjuízes}

O intuito foi verificar a concordância do diagnóstico entre Enfermeiro e Cirurgião-dentista e definir a possibilidade de uso destes instrumentos por profissionais graduados em Enfermagem para registro das condições de saúde e higiene bucal dos idosos que estão sob seus cuidados.

\section{Análise dos dados: tratamento estatístico}

A determinação da consistência interna foi verificada por meio da utilização do Coeficiente Alfa de Cronbach e a Análise de variância simples (ANOVA), servindo de indicadores de consistência interna do instrumento em estudo.

Para a determinação da estabilidade e confiabilidade foram considerados o percentual de concordância e o teste estatístico de Kappa, para análise das categorias individualmente, e teste de correlação para o escore total, tanto para profissionais entre si, quanto para profissional de enfermagem versus cirurgião-dentista.

Para o teste de correlação, após as análises das categorias individualmente, foram avaliados os escores totais dos exames. Para tanto, foi necessária uma reclassificação de referência transformando o tipo de variável (de discreta para categórica). Definiu-se então: Nível 1 para escores totais de 0 a 5; Nível 2 para escores totais de 6 a 10; Nível 3 para escores totais de 11 a 16.

Para interpretação do teste Kappa, foram considerados os seguintes valores de concordância: 0: Pobre; 0 a 0,20: Leve; 0,21 a 0,40: Considerável; 0,41 a 0,60: Moderada; 0,61 a 0,80: Substancial; 0,81 a 1: Excelente.(18) 


\section{Aspectos Éticos}

O projeto referente a esta pesquisa foi submetido ao Comitê de Ética em Pesquisa em Seres Humanos da Universidade Federal de Santa Catarina e aprovado segundo parecer 107/08. Todos os participantes foram informados sobre o objetivo e procedimentos da pesquisa e assinaram o Termo de Consentimento Livre e Esclarecido.

\section{RESULTADOS}

A Figura 1 apresenta o cruzamento comparativo dos escores emitidos por Cirurgião-dentista e Enfermeiro, segundo cada dimensão do instrumento ASBTO.

A Tabela 1 apresenta os valores de Kappa para os exames realizados pelo Enfermeiro e Cirurgião-dentista, calculados inicialmente para cada categoria do instrumento e sua classificação correspondente.
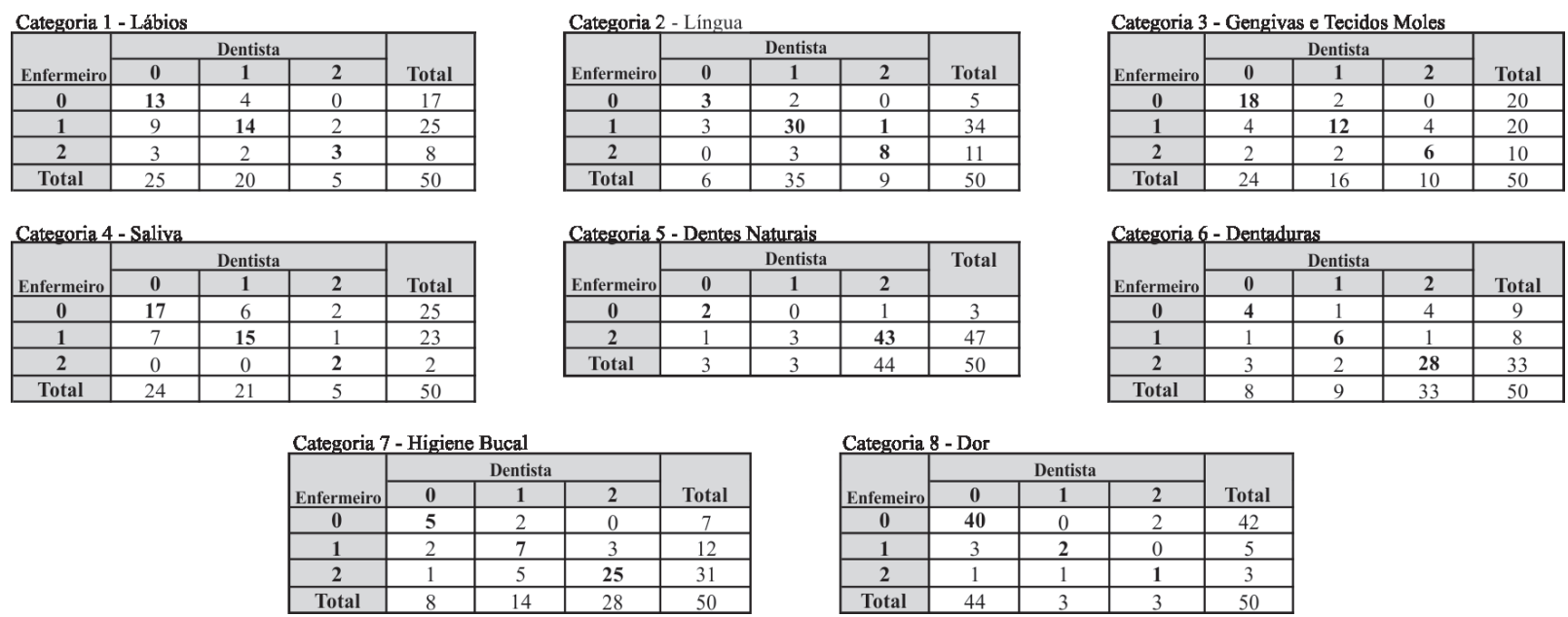

Figura 1 - Comparação dos escores emitidos por Enfermeiro e Cirurgião-dentista por Categoria do instrumento ASBTO. Florianópolis, SC, 2010.

Tabela 1 - Valores do coeficiente Kappa para os exames realizados por Enfermeiro e Cirurgião-dentista, desvio padrão, intervalo de confiança e respectiva classificação, por categorias do instrumento ASBTO. Florianópolis, SC, 2010.

Intervalo de confiança

\begin{tabular}{|c|c|c|c|}
\hline $\begin{array}{l}\text { Kappa } \\
\text { Observado }\end{array}$ & $\begin{array}{l}\text { Desvio } \\
\text { Padrão }\end{array}$ & $\begin{array}{l}\text { Limite } \\
\text { Inferior }\end{array}$ & $\begin{array}{l}\text { Limite } \\
\text { Superior }\end{array}$ \\
\hline
\end{tabular}

\begin{tabular}{lccccl}
\hline Categoria 1 - Lábios & 0,36 & 0,11 & 0,15 & 0,58 & Considerável \\
Categoria 2 - Língua & 0,65 & 0,10 & 0,44 & 0,85 & Substancial \\
Categoria 3 - Gengivas e & 0,60 & 0,09 & 0,42 & 0,72 & Substancial \\
tecidos moles & 0,43 & 0,12 & 0,18 & 0,67 & Moderado \\
Categoria 4 - Saliva & 0,22 & 0,65 & 0,92 & Moderado \\
Categoria 5 - Dentes naturais & 0,49 & 0,12 & 0,23 & 0,72 & Moderado \\
Categoria 6 - Dentaduras & 0,48 & 0,09 & 0,43 & 0,80 & Substancial \\
Categoria 7 - Higiene Bucal & 0,62 & 0,17 & 0,09 & 0,77 & Moderado \\
Categoria 8 - Dor & 0,43 & & & \\
\hline
\end{tabular}


Na categoria 1 (Lábios) houve concordância em 30 dos 50 exames realizados (60\%). O valor kappa atingiu 0,36. A maior diferença foi observada no momento em que o Enfermeiro assinalou código 1 (rachados, avermelhados nas comissuras e secos) ou 2 (presença de úlcera e sangramento) e o Dentista código o (lisos, rosados, úmidos), demonstrando uma tendência do dentista a classificar melhor a condição dos lábios. O ressecamento bucal e, conseqüentemente, o labial, é comum nas pessoas de idade avançada devido à rotineira ingestão de medicamentos que produzem como efeito colateral a diminuição do fluxo salivar. Pode ter havido variação das condições dos lábios dos idosos por alterações de temperatura e hidratação no momento do exame. Entretanto, a diferença parece residir no parâmetro adotado como "normal" por cada profissional e aponta a necessidade de treinamento intensivo previamente à aplicação do instrumento, especialmente no reconhecimento de condições bucais características do idoso.

Na categoria 2 (Língua) foi possível observar a igualdade de respostas em 41 das 50 aplicações dos exames, ou seja, $82 \%$ das resposta do Enfermeiro e do dentista foram coincidentes. O Kappa de 0,65 comprovou uma boa sintonia na realização dos exames neste quesito.

Na categoria 3 (Gengiva e tecidos), 36 dos 50 exames foram respondidos igualmente, o que corresponde a $72 \%$ de concordância, demonstrando assim uma boa estabilidade na visualização desta categoria. O valor Kappa atingiu 0,60.

$\mathrm{Na}$ categoria 4 (Saliva), 68\%, ou seja, 34 das 50 respostas foram concordantes. O valor Kappa observado foi de 0,43. Este resultado deve-se, principalmente, pela observação de 6 situações em que o Enfermeiro marcou o escore o (tecidos úmidos, salivação aquosa, fluxo livre desimpedido, sem obstrução) e o dentista marcou o escore 1 (tecidos secos e pagajosos, presença de pouca saliva). Por ser o exame sialométrico visual um procedimento mais complexo para a realização por um profissional que não foi formado na área odontológica e também pelos mesmos motivos já mencionados na análise da categoria 1 (desidratação temporária por uso de medicamentos no período de realização do exame) observou-se esta variação na Categoria 4.

$\mathrm{Na}$ categoria 5 (Dentes naturais), houve similaridade em $90 \%$ das respostas, e um kappa 0,43. Apesar da alta taxa de concordância, o kappa exibiu valor pequeno, pois os índices de acerto estavam concentrados apenas no escore 2, porém, baixos nos outros escores, o que pode ter influenciado os resultados.

Na categoria 6 (Dentaduras), que analisa o uso e a qualidade de próteses dentais removíveis, obtivemos uma concordância em $76 \%$ das respostas. Assim, 38 dos 50 exames foram preenchidos de forma idêntica para esta categoria. Entretanto, o kappa apresentou valor de 0,48. A queda dos valores de kappa deve-se a discrepância em alguns resultados nos quais há variações muito grandes nos exames, embora em pequenos números. Um dos fatores que pode ter gerado esta alteração é o desconforto que alguns profissionais, que não da área da odontologia, podem ter em manusear as próteses dentárias. Orientações sobre o manuseio de próteses removíveis, reforçando aspectos de biossegurança, podem, sem maiores complicações, vir a superar este empecilho.

A categoria 7 (Higiene bucal) obteve percentual de concordância de $74 \%$ e um kappa de 0,62. Esta categoria apesar de sua condição de variabilidade no dia-a-dia dos idosos obteve um bom indicador de confiabilidade neste instrumento. As divergências de respostas, apesar do pequeno número, devem-se provavelmente a esta variabilidade.

Pode-se observar pela análise dos dados da categoria 8 (Dor) boa concordância nas respostas, que correspondem a $86 \%$ do total, ou seja 43 dos 50 exames. Porém o kappa apresentou valor de 0,43. Esta categoria pode ter seus escores induzidos não pela destreza do profissional examinador, mas, pela variabilidade na resposta do idoso, visto que este é o único item resultante de um questionamento. Esta categoria pode também, tornar-se instável em alguns momentos, já que a dor é uma experiência sensitiva emocional, subjetiva, na qual os episódios podem sofrer oscilações. Este fato explicaria os resultados em que há variação de resposta de 2 para 0.

A distribuição da soma dos escores finais do instrumento ASBTO, atribuídos por enfermeiro e dentista, ocorreu da seguinte forma: no Nível 1 foram situados 11 idosos pelo dentista e 6 pelo enfermeiro, no Nível 2 foram situados 29 idosos pelo dentista e 32 pelo enfermeiro e no Nível 3, 10 pelo dentista e 12 pelo enfermeiro. Após reclassificação dos escores totais finais nestes Níveis, a concordância entre Enfermeiro e Dentista alcançou percentual de 66\% (33 dos 50 escores possíveis foram coincidentes nos Níveis 1, 2 e 3). As diferenças de escores, se modestas, não influenciam no resultado final do 
teste, que é a decisão de encaminhamento ou não para a assistência odontológica especializada. Isto porque todos os idosos assinalados com categorias 1 ou 2 devem ser encaminhados para o profissional Cirurgião-dentista. Vale ressaltar que este instrumento foi formulado originalmente num contexto de país desenvolvido, onde os idosos possuem uma condição epidemiológica de saúde bucal diferenciada dos brasileiros. Assim, o instrumento executa uma triagem rigorosa em relação a situações em que deve se proceder a encaminhamento. Esta situação é evidenciada devido ao fato de $100 \%$ dos participantes apresentarem escores maiores que 1 nos exames realizados por ambos os profissionais. Ou seja, no universo estudado, todos os idosos necessitariam de referência a assistência odontológica.

O valor de kappa total do instrumento ASBTO, considerando a reclassificação em Níveis 1, 2 ou 3, alcançou 0,46 com desvio padrão de 0,10 e intervalo de confiança a 95\% com limite inferior de 0,25 e limite superior de 0,67 .

Para a determinação da consistência interna foi utilizado o teste Alfa de Cronbach. Analisou-se a coerência entre os resultados das categorias entre si para cada examinador, Enfermeiro e Dentista. Os resultados podem ser visualizados nas Tabelas 2 e 3.

Tabela 2 - Resultado do teste Alfa de Cronbach: instrumentos aplicados pelo Enfermeiro $(\mathrm{n}=50)$. Florianópolis, SC, 2010.

\begin{tabular}{|c|c|c|c|c|c|}
\hline & Média & Variância & $\begin{array}{l}\text { Desvio } \\
\text { padrão }\end{array}$ & $\begin{array}{l}\text { Correlação } \\
\text { total }\end{array}$ & $\begin{array}{l}\text { Alfa de } \\
\text { Cronbach }\end{array}$ \\
\hline Categoria 1 - Lábios & 7,52 & 4,04 & 2,01 & 0,22 & 0,34 \\
\hline Categoria 2 - Língua & 7,22 & 4,49 & 2,11 & 0,14 & 0,38 \\
\hline $\begin{array}{l}\text { Categoria } 3-\text { Gengivas e } \\
\text { tecidos moles }\end{array}$ & 7,54 & 3,48 & 1,86 & 0,39 & 0,24 \\
\hline Categoria $4-$ Saliva & 7,80 & 4,68 & 2,16 & 0,05 & 0,42 \\
\hline Categoria 5 - Dentes naturais & 6,46 & 4,72 & 2,17 & 0,09 & 0,40 \\
\hline Categoria 6 -Dentaduras & 6,86 & 4,00 & 2,00 & 0,17 & 0,37 \\
\hline Categoria 7 - Higiene Bucal & 6,86 & 3,92 & 1,98 & 0,24 & 0,33 \\
\hline Categoria 8 - Dor & 8,12 & 4,82 & 2,19 & 0,01 & 0,43 \\
\hline
\end{tabular}

Legenda da escala: Média $=8,34 ;$ Desvio padrão $=2,29 ;$ Alfa de Cronbach $=0,40$

Tabela 3 - Resultado do teste Alfa de Cronbach: instrumentos aplicados pelo Cirurgião-dentista $(\mathrm{n}=50)$. Florianópolis, SC, 2010.

\begin{tabular}{llllll}
\hline & Média & Variância & $\begin{array}{l}\text { Desvio } \\
\text { padrão }\end{array}$ & $\begin{array}{l}\text { Correlação } \\
\text { total }\end{array}$ & $\begin{array}{l}\text { Alfa de } \\
\text { Cronbach }\end{array}$ \\
\hline Categoria 1 - Lábios & 7,32 & 5,69 & 2,38 & 0,42 & 0,54 \\
Categoria 2 - Língua & 6,84 & 6,25 & 2,50 & 0,29 & 0,58 \\
Categoria 3 - Gengivas e & 7,20 & 5,12 & 2,26 & 0,50 & 0,50 \\
tecidos moles & 7,30 & 5,45 & 2,33 & 0,51 & 0,51 \\
Categoria 4 - Saliva & 6,10 & 7,05 & 2,65 & 0,05 & 0,63 \\
Categoria 5 - Dentes naturais & 6,42 & 6,60 & 2,57 & 0,08 & 0,65 \\
Categoria 6 - Dentaduras & 6,52 & 5,41 & 2,32 & 0,43 & 0,53 \\
Categoria 7 - Higiene Bucal & 7,74 & 6,75 & 2,60 & 0,16 & 0,61 \\
Categoria 8 - Dor & & & & & \\
\hline
\end{tabular}


$\mathrm{Na}$ análise dos escores das categorias atribuídos pelo Enfermeiro encontram-se valores que indicam fraca correlação. Os menores valores encontrados foram os das categorias 3 (Gengiva e tecidos moles), e 7 (Higiene bucal) e 1 (Lábios) e os maiores valores os das categorias 8 (Dor) e 4 (Saliva). O valor médio de Alfa de Cronbach igual a 0,40.

$\mathrm{Na}$ análise dos resultados encontrados pelo Dentista os menores valores foram achados nas categorias 3 (Gengivas e tecidos) e 4 (Saliva), o que coincide, em parte, com a do Enfermeiro. Já o valor médio do Alfa de Cronbach foi de 0,60. Por se tratar de um profissional formado na área específica, é natural que a consistência entre os itens seja maior quando comparada a profissionais de outras áreas.

A análise da variância foi realizada com o teste ANOVA para avaliação também da consistência interna do instrumento ASBTO. Os resultados encontrados podem ser observados na Tabela 4. Não houve diferença estatisticamente significante, ao nível de $95 \%$, entre os grupos Enfermeiro e Dentista $(\mathrm{p}>0,05)$.

Tabela 4- Média, desvio-padrão e valor de p dos escores totais finais dos instrumentos ASBTO atribuídos por Enfermeiro e Dentista $(\mathrm{n}=50)$. Florianópolis, SC, 2010.

\begin{tabular}{lllll}
\hline & $\mathrm{N}$ & Média & $\mathrm{DP}$ & $\mathrm{p}$ \\
\hline Enfermeiro & 50 & 8,34 & 2,29 & \\
Dentista & 50 & 7,92 & 2,76 & 0,45 \\
\hline
\end{tabular}

\section{DISCUSSÃO}

A avaliação das condições de saúde geral e bucal dos idosos pelos cuidadores é o primeiro passo na definição de um protocolo de cuidado à saúde bucal para idosos em ILPI. A higiene bucal diária de dentes e próteses, das mucosas bucais e a hidratação oral fazem parte do rol de procedimentos que serão individualmente prescritos de acordo com as características e necessidades dos idosos. Assim também se inclui a avaliação da necessidade de encaminhamento para atendimento especializado pelo Cirurgião-dentista. Este que deve atuar como colaborador, acompanhando todo o processo. $\mathrm{O}$ dentista deve promover oportunidades e espaços para a capacitação teórica e o treinamento prático dos cuidadores, sensibilizando-os para a importância da avaliação das condições bucais, inserida num protocolo abrangente de ações de cuidado à saúde bucal dos idosos ${ }^{(2,19)}$.

A saúde bucal é componente inseparável do sistema de saúde corporal, capaz de afetar além do bem-estar físico, também o bem-estar psicossocial das pessoas. Assim, defende-se que o cuidado à saúde bucal também deve e necessita ser componente inseparável e articulado de qualquer sistema de atenção integral à saúde do idoso, e particularmente dos idosos institucionalizados. A organização do cuidado à saúde bucal no âmbito das ILPI deve estar integrada às demais atividades assistenciais de saúde, não perdendo a perspectiva da necessidade de ações mais amplas, como as de educação em saúde e preventivas.

É possível implementar tecnologias de cuidado leves, não exigindo muito dispêndio de tempo nem sobrecarregando os cuidadores. Para tanto, faz-se necessário preparo dos cuidadores para que possam aplicar instrumentos de diagnóstico e intervenção no contex to das ILPI ${ }^{(19,20)}$, visando à melhora e manutenção da sua saúde bucal.

Para a elaboração de um protocolo de cuidado à saúde bucal padronizado, mas flexível no que diz respeito às necessidades individuais de cada idoso residente, torna-se imperativo o conhecimento das condições de saúde bucal do idoso, incluindo dentes, próteses, tecidos moles, padrão salivar e presença de dor, necessidade de tratamento imediata e definição do rol de procedimentos necessários para que mantenha uma higiene bucal satisfatória que permita resgatar e manter sua saúde bucal. Desse modo, a coleta destas informações e registro desses dados demanda um instrumento validado, adequado à realidade sócio-cultural das instituições, dos profissionais de saúde e também dos idosos. ${ }^{(16,19)}$

$\mathrm{O}$ receio de não diagnosticar adequadamente algum agravo ou até mesmo deixar de observar alguma condição clínica importante, que fosse determinante para o encaminhamento do idoso à assistência odontológica pode ter contribuído para a tendência do examinador Enfermeiro, em algumas categorias, de superestimar, negativamente, a condição bucal do idoso.

O instrumento ASBTO é passível de ser utilizado por profissionais da área da Enfermagem para avaliação das condições de saúde bucal de idosos em ILPI e conseqüente encaminhamento. Entretanto, sugere-se que sua aplicação se dê em um contexto que inclua capacitação teórico-prática prévia e continuada, adequada às condições epidemiológicas bucais dos idosos brasileiros. 
A discussão de situações clínicas entre profissionais contribui para a uniformização dos exames e estabelecimento de parâmetros aceitáveis para os achados.

Sugere-se, para melhores resultados, tomar em conta as ponderações que foram apresentadas neste estudo, em cada uma das suas categorias de exame. Vale ressaltar alguns fatores que influenciam os cuidadores na provisão do cuidado à saúde bucal e que por vezes são impeditivos da adequada atenção à saúde bucal: a falta de tempo e de pessoal, dificuldades físicas e de comportamento dos idosos e a falta de um programa de educação em saúde bucal específico para cuidadores de idosos. Essas questões precisam ser tomadas em conta quando da implementação de instrumentos de avaliação das condições de saúde bucal, inseridos em protocolos de cuidado ao idoso residente em ILPI.

\section{CONCLUSÃo}

Em relação à determinação da estabilidade e confiabilidade do instrumento ASBTO, os percentuais de concordância entre Enfermeiro e Dentista variaram de $60 \%$, na categoria Lábio, até $90 \%$, na categoria Dentes naturais. $\mathrm{O}$ valor Kappa total do instrumento alcançou 0,46, sendo considerado moderado.

A consistência interna do ASBTO, quando aplicado pelo profissional Enfermeiro, revelou menores valores nas categorias Gengiva e tecidos moles, Lábio e Higiene bucal. Na análise dos resultados encontrados pelo Dentista, os menores valores encontraram-se nas categorias Gengivas e tecidos moles e Saliva. O valor médio do Alfa de Cronbach foi de 0,40 para Enfermeiro e 0,60 para Cirurgião-dentista. Não houve diferença estatística significante entre as médias finais do instrumento obtidas pelo Enfermeiro e Dentista $(\mathrm{p}=0,41)$.

Pode-se concluir que este instrumento é passível de ser utilizado por profissionais da área da Enfermagem para avaliação e rastreamento das condições de saúde bucal de idosos em ILPI. Sugere-se que esta utilização deva estar inserida num processo mais amplo que inclua capacitação teórica e prática de cuidadores. Faz-se necessário treinamento não só dos critérios do instrumento, mas da prática de examinar a cavidade bucal de idosos, que nem sempre constitui numa tarefa de fácil execução, principalmente quando em situação de fragilidade.

\section{REFERÊNCIAS}

1 Macentee MI. Missing links in oral health care for frail elderly people. J Can Dent Assoc. 2006;72(5):421-5.

2 De Visschere L, de Baat C, Schols JM, Deschepper E, Vanobbergen J. Evaluation of the implementation of an 'oral hygiene protocol' in nursing homes: a 5-year longitudinal study. Community Dent Oral Epidemiol. 2011;39(5):416-25.

3 De Visschere LM, Grooten L, Theuniers G., Vanobbergen JN. Oral hygiene of elderly people in long-term care institutions--a cross-sectional study. Gerodontology. 2006;23(4):195-204.

4. Montal S, Tramini P, Triay JA, Valcarcel J. Oral hygiene and the need for treatment of the dependent institutionalized elderly. Gerodontology. 2006;23(2):67-72.

5 Saliba NA, Moimaz SAS, Marques JA, Prado, RL. Perfil de cuidadores de idosos e percepção sobre saúde bucal. Interface 2007;1 1(21):39-50.

6 Peltola P, Vehkalahti MM, Wuolijoki-Saaristo K. Oral health and treatment needs of the long-term hospitalized elderly. Gerontology. 2004;2 1(2):93-9.

7 Brasil. Ministério da Saúde. Secretaria de Vigilância em Saúde. Secretaria de Assistência à Saúde. Departamento de Atenção Básica. Coordenação Nacional de Saúde Bucal. Pesquisa Nacional de Saúde Bucal - 2010. Nota para a imprensa. Brasília: Ministério da Saúde; 2010.

8 Chalmers JM, Pearson A. A systematic review of oral health assessment by nurses and carers for residents with dementia in residential care facilities. Spec Care Dentist. 2005; 25(5):227-33.

9 Atalaia-Silva KC, Lourenço RA. Tradução, adaptação e validação de construto do Teste do Relógio aplicado entre idosos no Brasil. Rev Saúde Públ. 2008;42(5):930-7.

10 Almeida MHM, Spínola AWP, Iwamizu PS, Okura RIS, Barroso LP, Lima ACP. Confiabilidade do instrumento para classificação de idosos quanto à capacidade para o autocuidado. Rev Saúde Públ. 2008;42(2):317-23.

11 Minosso JSM, Amendola F, Alvarenga MRM, Oliveira MAC. Validação, no Brasil, do Índice de Barthel em idosos atendidos em ambulatórios. Acta Paul Enferm. 2010;23(2):218-23. 
12 Ferrari SC, Santos FC, Guarnieri AP, Salvador N, Correa AZH, Hala AZA et al. Índice Manchester de incapacidade associada ao pé doloroso no idoso: tradução, adaptação cultural e validação para a língua portuguesa. Rev Bras Reumatol. 2008;48(6):335-41.

13 Petreça DR, Benedetti TRB, Silva DAS. Validação do teste de flexibilidade da AAHPERD para idosos Brasileiros. Rev Bras Cineantropom Desempenho Hum. 201 1; 1(6):455-60.

14 Dias IB, Carvalho PG. A qualidade percebida em programas municipais de actividade física para idosos: validação estatística para Portugal. Motriz: Rev Educ Fis. 2011; 17(1):145-59.

15 Urrutia CG, Santander IE, Ormazábal FR. Spanish translation and validation of the nursing dental coping beliefs scale. Rev Esp Salud Publica. 2010;84(4):409-16.
16 Chalmers JM, King PL, Spencer AJ, Wright FA, Carter KD. The oral health assessment tool-validity and reliability. Aust Dent J. 2005;50(3):191-9.

17 Gonçalves LHT, Mello ALSF, Zimermann K. Validação de instrumento de avaliação das condições de saúde bucal de idosos institucionalizados. Esc Anna Nery. 2010;14(4): 839-47.

18 Cerda L J, Villarroel L. Evaluación de la concordancia inter-observador en investigación pediátrica: Coeficiente de Kappa. Rev Chil Pediatr. 2008;79(1):54-8.

19 Gil-Montoya JA, De Mello AL, Cardenas CB, Lopez IG. Oral health protocol for the dependent institutionalized elderly. Geriatr Nurs. 2006; 27(2):95-101.

20 Jesus IS, Sena ELS, Meira EC, Gonçalves LHT, Alvarez AM. Cuidado sistematizado a idosos com afeccão demencial residentes em instituição de longa permanência. Rev Gaúcha Enferm. 2010;31(2): 285-92.

\author{
Endereço do autor / Dirección del autor / \\ Author's address: \\ Ana Lúcia S. Ferreira de Mello \\ Rua Rosa, 159, Pantanal \\ 88040-270, Florianópolis, SC \\ E-mail: alfm@,terra.com.br \\ Telefone: 5548 3721-5144
}

